\title{
Effects of Filgrastim on Thrombocytopenia in Patients with Dengue Fever: A Retrospective Study
}

\section{Deepak D Baid and Vivika Jain \\ Nulife Hospital, Ghatkopar West, Mumbai, India}

*Corresponding author: Dr. Deepak D Baid, NULIFE Hospital, A1-HareKrishna, L.B.S Marg, Ghatkopar (W), Mumbai-86, Email: dr.deepakbaid@gmail.com

\section{Research Article}

Volume 2 Issue 7

Received Date: June 01, 2018

Published Date: June 15, 2018

\section{Abstract}

Background: Dengue infection causes hematological disturbances including agranulocytosis, leucopenia, and thrombocytopenia. Platelet transfusion is generally used for hematological corrections, but they are not always useful, and are expensive too. Filgrastim is effective in mobilizing the progenitor cells to stimulate the production of platelets and white blood cells (WBCs) endogenously.

Aims: This observational, retrospective, single-center study aimed to evaluate the effects of filgrastim in improving platelet and WBC counts in patients with dengue fever.

Methods: Data of patients with dengue fever admitted to Nulife Hospital, Mumbai, who had received filgrastim (Neukine ${ }^{\circledR}$ ), were analyzed. Dengue was diagnosed by the rapid dengue NS1 antigen and dengue IgM/IgG antibody tests. Patients with severe thrombocytopenia (platelet count : $<20000 / \mathrm{mm}^{3}$ with or without bleeding) were given a single subcutaneous dose of filgrastim $300 \mu \mathrm{g} / \mathrm{mL}$. Patients with dengue hemorrhagic fever and dengue shock syndrome were excluded.

Results: Data of 51 patients with dengue fever with thrombocytopenia, or dengue like fever with thrombocytopenia (patients in whom reduction in fever followed by leucocytes and platelets were observed) analyzed. Majority (56.86\%) had severe thrombocytopenia (platelet count: $<20000 / \mathrm{mm}^{3}$ ) at baseline. A significant increase $(\mathrm{p}<0.001)$ was observed in the platelet and WBC counts on Days 1 and 2 after filgrastim administration. Doubling of the platelet count was seen in 41 patients within 2 days, and WBC within a day post filgrastim use. Maximum increase ( 800\%) in platelet count was seen at discharge in patients with baseline platelet count $<10000 / \mathrm{mm}^{3}$. Proportion of patients receiving platelet transfusion was lower after filgrastim use than before its administration (9 vs. 22). Filgrastim use was also associated with improvement in the fever status in majority of patients. None of the patients reported any complications after filgrastim use. Liver, renal and hematological evaluations did not show any adverse effects. The follow-up evaluation post discharge was normalization of hematological parameters (as per the clinician), but did not form part of this study.

Conclusion: This is the first study to demonstrate that filgrastim use persistently increases the platelet counts along with white blood cell counts within a day in patients with dengue. Addition of filgrastim to the treatment of dengue may help 
in reducing the transfusion requirement and fever episodes, and thus improving the disease outcome. More studies will be required to analyze if there is reduction in bleeding tendency and to confirm the disease outcomes.

Keywords: Dengue; Fever; Thrombocytopenia; Platelet; White Blood Cell; Filgrastim; Neukine ${ }^{\circledR}$

\section{Introduction}

Dengue is a mosquito-borne viral infection transmitted in humans by Aedesaegypti in the form of four distinct serotypes (DENV-1, DENV-2, DENV-3, and DENV-4) [1-3], causing the disease ranging from asymptomatic infection to undifferentiated fever, dengue fever and dengue hemorrhagic fever [2,4]. It is the most important acute systemic arthropod-borne viral infection in humans [5]. Currently an expanding global problem, dengue is endemic from tropical regions to most subtropical regions of the world including Asia, the Pacific, the Americas, Africa, and the Caribbean, causing human suffering and massive socioeconomic losses [4,6]. The Center for Disease Control estimates that $\sim 40 \%$ of world's population live in dengue risk areas, and the World Health Organization estimates that 50 to 100 million dengue infections occur yearly, including 500,000 dengue hemorrhagic fever cases and 22,000 deaths, mostly among children [6]. Dengue is endemic in India with an estimated $\sim 33$ million apparent and $\sim 100$ million asymptomatic infections in 2010, which were the largest number of dengue cases globally [7]. According to the National Vector Borne Disease Control Programme of India, 74,454 dengue cases with 167 deaths were reported in 2013 [8].

Pathogenesis of dengue involves suppression of the immune system leading to the characteristic presentation of hematological disturbances including thrombocytopenia, agranulocytosis or leucopenia, hypovolemia, hypotension, increased vascular permeability, or shock $[9,10]$. Dengue fever is associated with leucopenia, neutropenia, relative lymphocytosis and increased atypical lymphocytes [10]. A study in 543 cases with dengue infection demonstrated that leucopenia, thrombocytopenia, lymphocytopenia and atypical lymphocytes were the main hematological findings [11]. Thrombocytopenia is the most common hematological finding in patients with dengue infection. It is caused due to IgM antiplatelet antibodies, which induce platelet lysis via complement activation $[3,12]$. It was stated by Kharat and Kulkarni that dengue patients with a bleeding tendency and/or a platelet count $<25000 / \mathrm{mm}^{3}$ may be transfused platelets since there is no specific therapy available [12]. Platelet transfusion can prevent bleeding, but infectious and allergic complications and refractoriness due to alloimmunization may occur [3]. Platelet transfusion is not always beneficial, and certain races religiously disallow administration of blood and blood products [13]. Furthermore, a study conducted by Indian researchers showed that in $\sim 50 \%$ of patients with dengue with thrombocytopenia, platelet transfusion did not prevent development of severe bleeding or shorten the time to cessation of bleeding [14]. Stimulation of platelet production may help in the treatment of thrombocytopenia associated with dengue [15].

Granulocyte colony-stimulating factor (G-CSF) is a hematopoietic hormone that can mobilize committed progenitor cells, and increase the peripheral white blood cell (WBC) count, absolute neutrophil count (ANC), lymphocytes and monocytes [16]. It also induces a multilineage response and mobilizes the myeloid, erythroid, megakaryocyte (platelet originating cells) and multipotential progenitor cells [17].

Filgrastim, a G-CSF, has been mentioned as a rescue drug in certain high risk patients with established febrile neutropenia (ANC $<100 / \mu \mathrm{L}$ ) [9]. Filgrastim has been shown to increase the numbers of granulocytemacrophage, and peripheral-blood progenitor cells leading to platelet recovery [18]. A transient increase in neutrophil counts is typically seen 1 to 2 days after initiation of filgrastim therapy [19]. Furthermore, improvements in the leucocyte and platelet counts were observed following filgrastim treatment in individual cases of patients with dengue infection $[9,10]$.

Aforementioned data suggests that filgrastim use can improve the platelet and WBC counts, which can potentially help patients with dengue infection. No prior study has specifically evaluated the use of filgrastim in these patients for the correction of blood cell counts, especially the platelet counts. Hence, the current study was an attempt to correct the hematological disturbances including platelet counts in dengue patients with the use of filgrastim. 


\section{Materials and Methods}

\section{Study Population}

Patients of all age groups and both sexes were included if they had a clinical diagnosis of dengue fever, treated with filgrastim, and whose baseline serum samples were collected within 1-7 days from the onset of symptoms that were consistent with dengue infection. Patients with dengue hemorrhagic fever and dengue shock syndrome were excluded. Dengue fever was confirmed by the laboratory tests (rapid dengue NS1 antigen and dengue IgG/IgM antibody tests). It was observed that many patients had low platelet and leucocyte counts, and the treatment resulted in improvements first in fever followed by the leucocytes and lastly the platelets. Patients who followed this trend (falling leucocytes and platelets with improvement of fever) with absent markers for dengue fever were labeled as "dengue like fever". Due to socioeconomic status of the patients, further testing for other viruses could not be done.

\section{Study Design}

This was an observational, retrospective, analysis in dengue patients who had received subcutaneous filgrastim $300 \mu \mathrm{g} / \mathrm{mL}$ (Neukine ${ }^{\circledR}$ by Intas Pharmaceuticals Ltd., Ahmedabad) between 1 January 2009 and 20 July 2016 in an inpatient setting at a single-center, Nulife Hospital, Mumbai, India. Most (84.31\%) of the patients received a single dose of filgrastim. The medical records of these patients were retrospectively analyzed for the effects of filgrastim on platelet and WBC counts as well as any adverse effects.

The study protocol was reviewed and approved by the independent 'Intersystem Biomedica Ethics Committee', Mumbai. The study was conducted in accordance with the ethical principles that have their origin in the Declaration of Helsinki, and in accordance with the International Conference on Harmonization's Good Clinical Practice guidelines, applicable regulatory requirements, and in compliance with the protocol.

\section{Study Endpoints}

The primary endpoint was the improvement in the platelet count at Day 2 after filgrastim administration. Secondary endpoints were the improvements in the WBC count at Day 2 after filgrastim administration and the proportion of patients who recovered from dengue infection and discharged.

\section{Statistical Analysis}

SAS version 9.3 (SAS Institute Inc., USA) was used for statistical analysis. Demographic and baseline characteristics were summarized descriptively. Categorical variables were summarized with frequency and percentage. Continuous variables were summarized with count, mean, standard deviation, median, minimum and maximum. Paired t-test was used to calculate the $\mathrm{P}$ values.

\section{Results}

\section{Patient Disposition and Demographics}

Data of 51 patients with dengue fever confirmed by the laboratory test (rapid dengue NS1 antigen and dengue IgG/IgM antibody tests) who were administered filgrastim between 1 January 2009 and 20 July 2016 were analyzed. Patients were diagnosed as dengue like fever with thrombocytopenia (62.75\%) or dengue with thrombocytopenia (37.25\%). Overall, $56.86 \%$ of the patients had severe thrombocytopenia (platelet count: $<20,000 / \mathrm{mm}^{3}$ ) and there were no signs of bleeding reported. The baseline characteristics of the patients are summarized in Table 1.

\begin{tabular}{|c|c|}
\hline Parameters & $\begin{array}{c}\text { Patients who received } \\
\text { filgrastim }(\mathrm{N}=51)\end{array}$ \\
\hline \multicolumn{2}{|l|}{ Age, n (\%) } \\
\hline $15-24$ years & $19(37.25)$ \\
\hline 25-34 years & $14(27.45)$ \\
\hline $35-44$ years & $12(23.53)$ \\
\hline 45-54 years & $3(5.88)$ \\
\hline $55+$ years & $3(5.88)$ \\
\hline \multicolumn{2}{|l|}{ Sex, n (\%) } \\
\hline Men & $38(74.51)$ \\
\hline Women & $13(25.49)$ \\
\hline \multicolumn{2}{|l|}{ Fever at baseline visit, n (\%) } \\
\hline No & $32(62.75)$ \\
\hline Yes & $19(37.25)$ \\
\hline \multicolumn{2}{|l|}{$\begin{array}{l}\text { Platelet counts at baseline } \\
\text { visit }\left(\text { per } \mathrm{mm}^{3}\right), \mathbf{n}(\%)\end{array}$} \\
\hline$<10,000$ & $18(35.29)$ \\
\hline $10001-20000$ & $11(21.57)$ \\
\hline $20001-30000$ & $8(15.69)$ \\
\hline $30001-50000$ & $6(11.76)$ \\
\hline $50000-100000$ & $3(5.88)$ \\
\hline$>100000$ & $5(9.80)$ \\
\hline \multicolumn{2}{|l|}{ Diagnosis, n (\%) } \\
\hline Dengue with thrombocytopenia & $19(37.25)$ \\
\hline $\begin{array}{l}\text { Dengue like fever with } \\
\text { thrombocytopenia }\end{array}$ & $32(62.75)$ \\
\hline
\end{tabular}

Table 1: Patient disposition and baseline characteristics. 


\section{Endpoints}

A consistent increase in the median platelet counts were observed after filgrastim administration from baseline $\left(14000 / \mathrm{mm}^{3}\right)$ to Day $2\left(46500 / \mathrm{mm}^{3}\right)$. Furthermore, increase in the platelet counts were also observed as early as Day $1\left(31000 / \mathrm{mm}^{3} ; \mathrm{p}<0.0001\right.$ at both time points) (Figure1). Similarly, a significant increase in the median WBC counts were observed from baseline $\left(4800 / \mathrm{mm}^{3}\right)$ to Day $2\left(20600 / \mathrm{mm}^{3}, \mathrm{p}<0.05\right)$. Increased WBC counts were also seen at Day 1 $\left(17800 / \mathrm{mm}^{3}, \mathrm{p}<0.0001\right)$ post filgrastim administration (Figure 2).
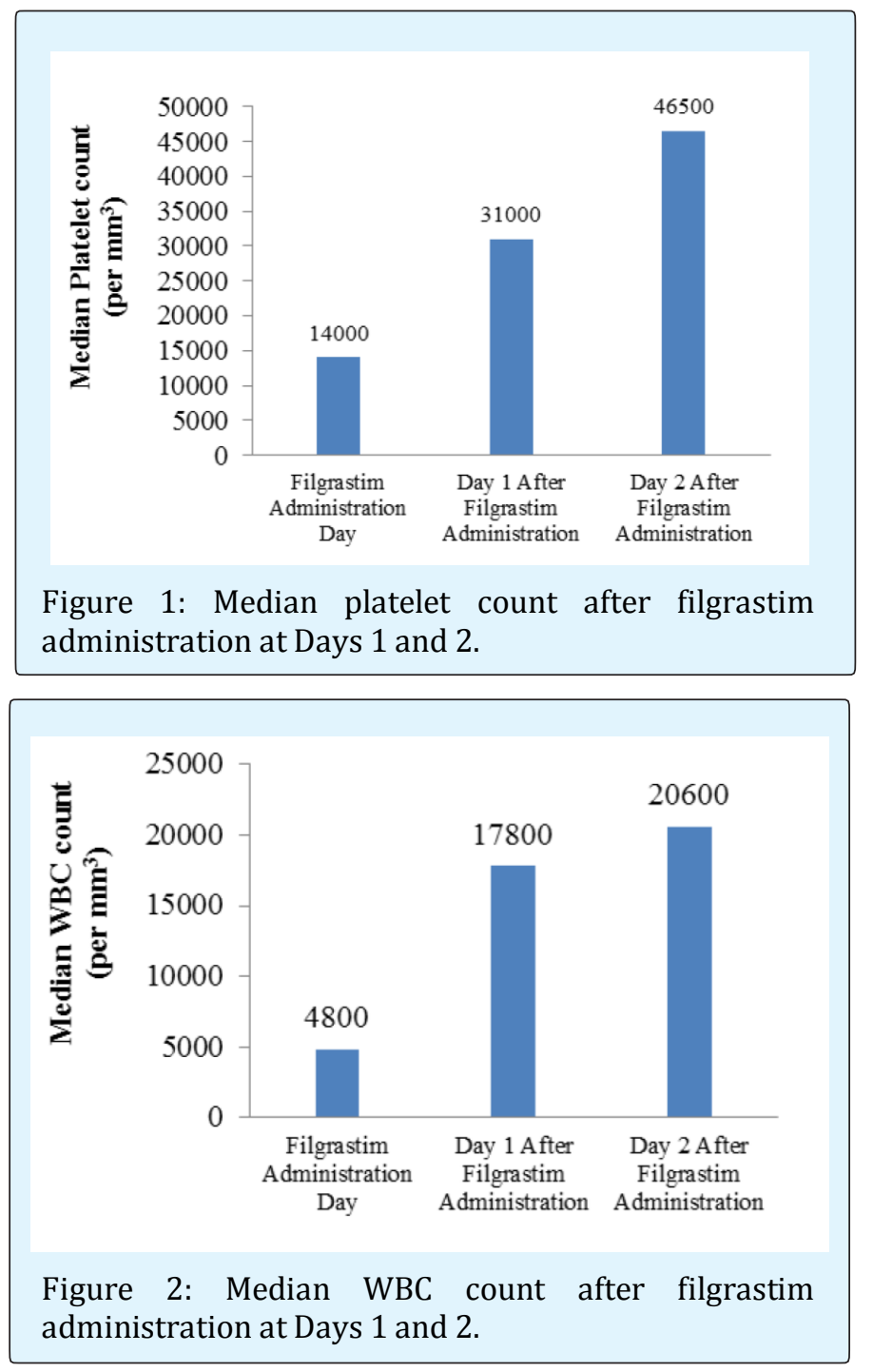

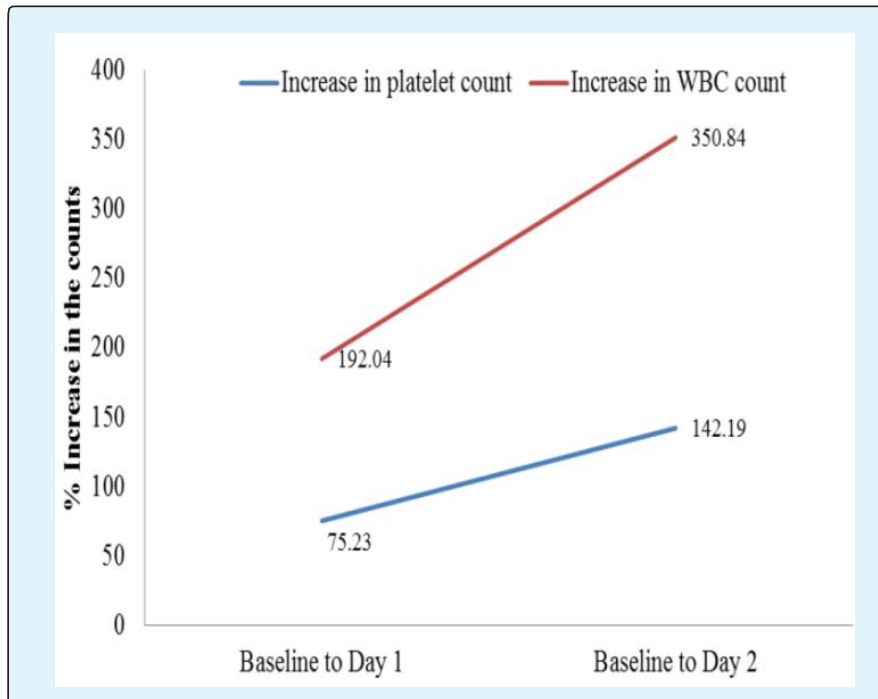

Figure 3: Correlation in the increase in platelet and WBC counts after filgrastim administration.

Overall, a platelet count of $\geq 50000 / \mathrm{mm}^{3}$ was achieved in 41 patients in a median of 3 days. Platelet counts were doubled from the values before filgrastim administration within a median of 2 days, and similarly the WBC counts were doubled in a median of 1 day. Correlation in the increase in platelet and WBC counts after filgrastim administration is shown in Figure 3, which indicate a higher increase in the WBC counts than the platelets at both Days 1 and 2 after filgrastim administration.

Median time to start filgrastim was 2 days after admission. Overall hospitalization period was 7 days in this study, and patients were discharged at an average of 5 days after filgrastim administration. Filgrastim was started in a majority of the patients who had a median baseline platelet count of $<20,000 / \mathrm{mm}^{3} \quad(n=32)$. After filgrastim administration, an increase in the median platelet count was observed at the time of discharge with $978 \%$ increase in patients with a baseline platelet count of $<10,000 / \mathrm{mm}^{3}$, and $422 \%$ in $10001-20000 / \mathrm{mm}^{3}$ (Table 2). 


\begin{tabular}{|c|c|c|c|c|}
\hline Platelet Count per $\mathbf{~ m m}^{\mathbf{3}}$ & $\mathbf{n}$ & $\begin{array}{c}\text { Median platelet count at } \\
\text { start per } \mathbf{~ m m}^{\mathbf{3}}\end{array}$ & $\begin{array}{c}\text { Median platelet count at } \\
\text { discharge per } \mathbf{~ m m}^{\mathbf{3}}\end{array}$ & Difference (\%) \\
\hline$<10000$ & 14 & 7000 & 75500 & 978.57 \\
\hline $10001-20000$ & 18 & 13500 & 70500 & 422.22 \\
\hline $20001-30000$ & 10 & 24500 & 66950 & 173.27 \\
\hline$>30000$ & 5 & 184000 & 88000 & 52.17 \\
\hline
\end{tabular}

Table 2: Criteria to start filgrastim based on the median platelet counts $\left(\mathrm{N}=47^{*}\right)$.

*Platelet counts missing for 4 patients before filgrastim administration and were not included in the analysis.

Overall, 22 patients received platelet transfusion before the administration of filgrastim with average packs administered at 7 (maximum 20 packs), and the median platelet and WBC counts in these patients were 17000 and $4450 / \mathrm{mm}^{3}$, respectively, before filgrastim use, and 28000 and $21150 / \mathrm{mm}^{3}$ after filgrastim use. Nine patients received platelet transfusion after the administration of filgrastim with the average number of packs administered at 4 (maximum 10 packs).

Overall 19 (median WBC count: $2800 / \mathrm{mm}^{3}$ ) of 51 patients had fever before the administration of filgrastim, which decreased to 6 patients (median WBC: $4600 / \mathrm{mm}^{3}$ ) having fever after filgrastim administration. The median WBC count was $17800 / \mathrm{mm}^{3}$ in 13 patients in whom fever resolved after filgrastim administration.

There was no adverse event reported related to filgrastim. The evaluation of liver, renal and hematological profile did not show any adverse effects. The follow-up evaluation post discharge was for the normalization of hematological parameters (as per the clinician), but did not form part of this study.

\section{Discussion}

Dengue infection has become a major public concern globally and it is the most important mosquito-borne disease in the Indian subcontinent, with frequent epidemic episodes occurring especially during monsoons $[20,21]$. Hematological abnormalities including leucopenia, thrombocytopenia, neutropenia, lymphocytosis, and hypovolemia, hypotension, increased vascular permeability, or shock are the characteristics of dengue infection. Thrombocytopenia is amongst the most common hematological complication of dengue infection, which may lead to severe conditions such as sudden bleeding or shock [22]. Platelet transfusion may be given to the patients who are at a high risk of bleeding and have severe thrombocytopenia. Makroo and colleagues demonstrated that high-risk patients having platelet count $<20,000 / \mathrm{mm}^{3}$ and risk of bleeding require urgent platelet transfusion [23]. Platelet transfusion has been shown to be effective in the prevention of bleeding, however, it is not always beneficial and it may lead to complications related to infection, allergy, or refractory bleeding. Furthermore, the administration of blood and blood products are prohibited in certain populations due to religious beliefs [13]. Hence, there is a need for agents which may increase endogenous platelet production. The current study has assessed the effects of filgrastim on the improvements in platelet and WBC counts.

Filgrastim, a G-CSF, promotes the growth and maturation of myeloid cells, and has been mentioned as a rescue drug in certain high risk patients with established febrile neutropenia [9]. The G-CSF alone can mobilize not only committed progenitors, such as colony-forming unitgranulocyte, macrophage, burst forming unit-erythroid and mix-colony-forming unit, but also long-term culture initiating cells into the peripheral blood of normal individuals. In a study, 26 healthy donors for allogeneic transplantation with age between 21-41 years were mobilized with G-CSF (filgrastim [n=13] or lenograstim [n=13]) at a dose of $7.5 \mu \mathrm{g} / \mathrm{kg} /$ day subcutaneously for 5 days. The G-CSF administration resulted in a 6-fold increase in the peripheral WBC count, 9-fold increase in the ANC, 2-fold increase in the absolute number of lymphocytes and 3-fold increase in the absolute number of mononuclear cells (lymphocytes and monocytes) on day 5 compared with the baseline values [16]. Long-term administration of recombinant G-CSF has shown to induce a multilineage response and mobilized myeloid, erythroid, megakaryocyte and multipotential progenitor cells [17]. Furthermore, Shimoda and others have shown that G-CSF receptors are present on the surface of platelets $[24,25]$. These receptors appear to be functional in vitro and in vivo in healthy volunteers and the activation of platelets may be beneficial in thrombocytopenia $[25,26]$.

Sheridan and colleagues assessed the ability of filgrastim on the recovery of neutrophil and platelet counts after chemotherapy. The results demonstrated that filgrastim treatment increased the numbers of granulocyte-macrophage progenitors in peripheral blood up to a median of 58-fold over pretreatment values. Furthermore, platelet recovery was significantly faster in 


\section{Virology \& Immunology Journal}

patients receiving chemotherapy in combination with filgrastim than in patients who received chemotherapy alone; the platelet counts reaching $50 \times 10^{9} / \mathrm{L}$ in a median of 15 days after infusion of hemopoietic cells in the study patients compared with 39 days in controls $(p=0.0006)$ [17]. Chaisiripoomkere and colleagues have suggested a decrease in platelet count [16], whereas several other clinical studies have indicated an increased platelet count with G-CSF (filgrastim) use $[27,28]$. There is a lack of evidence on the utility of filgrastim to improve platelet and WBC counts in patients with dengue fever, except for two published case reports $[9,10]$. To our knowledge, the current study is the first to evaluate the effects of filgrastim on platelet and WBC counts in patients with dengue. The results demonstrated a significant increase in the platelet and WBC counts after filgrastim use in these patients.

Overall, an increase in neutrophil counts is typically seen 1 to 2 days after initiation of filgrastim therapy [19]. A study in healthy volunteers has shown increases in the ANC within a day after subcutaneous administration of $300 \mathrm{mg}$ single dose of filgrastim [29]. Furthermore, a preclinical study in healthy adult alpacas has shown an increase in the WBC and neutrophil counts within 24 hours after filgrastim administration [30]. Improvements in the leucocyte and platelet counts were observed the next day after rescue treatment with filgrastim $(150 \mu \mathrm{g}$ intravenous for 2 days) in a patient of dengue hemorrhagic fever with acute respiratory distress syndrome and myocarditis [9]. Filgrastim (300 $\mu \mathrm{g}$ subcutaneous for 1 day) normalized the leukocyte counts in a 14-year Thai boy with grade I dengue hemorrhagic fever, who had developed agranulocytosis [10]. In the current study, majority of the patients had severe thrombocytopenia and all the patients were administered filgrastim as per the clinician's discretion. Overall, an increase in the platelet and WBC counts started from the first day of filgrastim administration, which is consistent with the above observations.

Filgrastim was started in the majority $(62.74 \%)$ of patients if they had a median platelet count $<20000 / \mathrm{mm}^{3}$ $\left(\sim 14000 / \mathrm{mm}^{3}\right)$. The maximum increase $(978 \%)$ in the median platelet counts were seen in patients $(14 / 51$, $27 \%$ ) with a baseline platelet count of $<10000 / \mathrm{mm}^{3}$, and $422 \%$ in patients with baseline platelet count of 10001$20000 / \mathrm{mm}^{3}$ before filgrastim use. The platelet counts doubled within 2 days and WBCs in 1 day, with 41 patients showing a platelet count of $>50000 / \mathrm{mm}^{3}$ within 3 days of filgrastim use. Filgrastim was started after an average of 2 days of hospital admission. The acute phase of fever usually lasts between 3 and 7 days in dengue patients [31]. In the current study, fever subsided in majority (68\%) of the patients with a median time of 2 days after filgrastim use.

In a study in adult patients with dengue by Lee, et al., platelet transfusion did not lead to reduction in the bleeding or recovery in platelet counts in those without bleeding who had a platelet count $<20,000 / \mathrm{mm}^{3}$. Furthermore, platelet transfusion may slowdown the recovery of platelet to levels $>50,000 / \mathrm{mm}^{3}$ and increase the hospitalization stay [32]. In the current study, platelet transfusion requirement decreased in patients receiving filgrastim (22 patients before filgrastim use [average 7 packs] vs. 9 patients after [average4 packs]), and higher platelet counts were seen in such patients.

In patients with dengue or dengue like fever there may be associated liver parenchymal damage leading to elevation in some liver function tests irrespective of the improvement in platelet counts or fever [33,34]. The use of filgrastim has not been associated with increased liver function tests or hepatotoxicity [35]. In our study, liver function tests were abnormal in some patients prior to the administration of filgrastim and later too, but this could be attributed to the disease condition and not due to filgrastim administration.

This was a retrospective study with a small sample size of 51 patients. Also, the data on the effects of filgrastim on other organs like liver and kidneys were documented without any abnormalities attributed to filgrastim. Though filgrastim has been shown to increase blood cells of all lineages, the evidence in dengue fever was available only in a few case reports. This is the first such study that evaluated effects of filgrastim in a group of patients with dengue infection. These results need to be further confirmed in larger populations in prospective, randomized, controlled clinical trials.

Retrospective nature of the study is the major limitation of this study, which has observed the treatment outcomes without prespecified protocol, thus, there may be absence of data on potential confounding factors as the data was recorded in the past. Also, platelet transfusion was performed in some patients and prospective trials without transfusion may give a clearer picture to confirm the results of this study.

\section{Conclusion}

Though the complications of dengue infection have been mainly attributed to capillary leakage, there is a strong correlation to complications and platelet counts. 
The results of platelet transfusion have been nonrewarding and at times associated with its complications. Filgrastim may provide a useful option for patients with dengue who have low platelet counts. This is the first study to demonstrate that filgrastim use persistently increases the platelet and white blood cell counts within a day in patients with dengue and also reduces the duration of fever. The results need to be confirmed in larger, prospective, randomized studies.

\section{Funding}

The authors did not receive any external funding.

\section{References}

1. Cucunawangsih, Lugito NPH (2017) Trends of Dengue Disease Epidemiology. Virology (Auckl) 8: $1178122 \times 17695836$.

2. Thisyakorn U, Thisyakorn C (2015) Dengue: Global Threat. Southeast Asian J Trop Med Public Health 46(1): 3-10.

3. deAzeredo EL, Monteiro RQ, de-Oliveira Pinto LM (2015) Thrombocytopenia in Dengue: Interrelationship between Virus and the Imbalance between Coagulation and Fibrinolysis and Inflammatory Mediators. Mediators Inflamm 2015: 313842.

4. Gurugama P, Garg P, Perera J, Wijewickrama A, Seneviratne SL (2010) Dengue Viral Infections. Indian J Dermatol 55(1): 68-78.

5. Roth A, Mercier A, Lepers C, Hoy D, Duituturaga S, et al. (2014) Concurrent outbreaks of dengue, chikungunya and Zika virus infections - an unprecedented epidemic wave of mosquito-borne viruses in the Pacific 2012-2014. Euro Surveill 19(41): 20929.

6. Centers for Disease Control and Prevention (CDC) Dengue Epidemiology.

7. Bhatt S, Gething PW, Brady OJ, Messina JP, Farlow AW, et al. (2013) The global distribution and burden of dengue. Nature 496: 504-507.

8. Cecilia D (2014) Current status of dengue and chikungunya in India. WHO South-East Asia J Public Health 3(1): 22-27.
9. Deepak D, Garg R, Pawar M, Banerjee N, Solanki R, et al. (2011) Filgrastim as a Rescue Therapy for Persistent Neutropenia in a Case of Dengue Hemorrhagic Fever with Acute Respiratory Distress Syndrome and Myocarditis. Case Rep Anesthesiol 2011: 896783.

10. Insiripong S (2010) Agranulocytosis in dengue hemorrhagic fever: a neglected condition. J Med Assoc Thai 93(4): 502-504.

11. Oliveira EC, Pontes ER, Cunha RV, Froes B, Nascimento Dd (2009) Hematological abnormalities in patients with dengue. Rev Soc Bras Med Trop 42(6): 682-685.

12. Kharat N, Kulkarni R (2016) A study of clinical profile of patients with dengue fever with thrombocytopenia authors. WCT's Journal of Medicine 1: 1-6.

13. Chand NK, Subramanya HB, Rao GV (2014) Management of patients who refuse blood transfusion. Indian J Anaesth 58(5): 658-664.

14. Assir MZK, Kamran U, Ahmad HI, Basir S, Mansoor H, et al. (2013) Effectiveness of Platelet Transfusion in Dengue Fever: A Randomized Controlled Trial. Transfus Med Hemother 40(5): 362-368.

15. Fanucchi M, Glaspy J, Crawford J, Grast J, Figlin R, et al. (1997) Effects of polyethylene glycol-conjugated recombinant human megakaryocyte growth and development factor on platelet counts after chemotherapy for lung cancer. N Eng J Med 336(6): 404-409.

16. Chaisiripoomkere W, Jootar S, Ungkanont A (2001) Effect of G-CSF on peripheral blood progenitor cell mobilization and collection from healthy donors. Asian Pac J Allergy Immunol 19(3): 183-190.

17. Sonoda Y, Ohno Y, Fujii H, Takahashi T, Nakayama S, et al. (1993) Multilineage response in aplastic anemia patients following long-term administration of filgrastim (recombinant human granulocyte colony stimulating factor). Stem Cells 11(6): 543-554.

18. Sheridan WP, Begley CG, Juttner CA, Szer J, To LB, et al. (1992) Effect of peripheral-blood progenitor cells mobilised by filgrastim (G-CSF) on platelet recovery after high-dose chemotherapy. The Lancet 339(8794): 640-644. 


\section{Virology \& Immunology Journal}

19. Neupogen ${ }^{\circledR}$ Product Monograph.The US Food and Drug Administration (FDA).

20. Choudhary S, Shivkumar BR, Shankar A, Manjunatha YA, Priyadarshini MM (2013) Haematological Changes in Dengue Fever. National Journal of Basic Medical Sciences 3(4): 289-293.

21. Patel PM, Patel SK, Sabalpara MA, Shah CK, Shah NR (2016) Study of hematological and biochemical changes in dengue fever at tertiary care hospital at Ahmedabad. Int J Med Sci Public Health 5(9): 19341936.

22. Narayanan M, Aravind M, Ambikapathy P, Prema P, Jeyapaul MP (2003) Dengue fever-clinical and laboratory parameters associated with complications. WHO Regional Office for South-East Asia. Dengue Bulletin 27: 108-115.

23. Makroo RN, Raina V, Kumar P, Kanth RK (2007) Role of platelet transfusion in the management of dengue patients in a tertiary care hospital. Asian J Transfus Sci 1(1): 4-7.

24. Kanaji T, Okamura T, Nagafuji K, Iwasaki H, Shimoda $\mathrm{K}$, et al. (1995) Megakaryocytes produce the receptor for granulocyte colony-stimulating factor. Blood 85(11): 3359-3360.

25. Shimoda K, Okamura S, Harada N, Kondo S, Okamura $\mathrm{T}$, et al. (1993) Identification of a functional receptor for granulocyte colony-stimulating factor on platelets. J Clin Invest 91(4): 1310-1313.

26. Avenarius HJ, Freund M, Deinhardt J, Poliwode H (1992) Effect of recombinant human granulocyte colony-stimulating factor (rhG-CSF) on circulating platelets. Ann Hematol 65(1): 6-9.

27. Chao NJ, Schriber JR, Grimes K, Long GD, Negrin RS, et al. (1993) Granulocyte colony-stimulating factor" mobilized" peripheral blood progenitor cells accelerate granulocyte and platelet recovery after high-dose chemotherapy. Blood 81(8): 2031-2035.
28. Olivieri A, Scortechini I, Capelli D, Montanari M, Lucesole M, et al. (2004) Combined administration of alpha-erythropoietin and filgrastim can improve the outcome and cost balance of autologous stem cell transplantation in patients with lymphoproliferative disorders. Bone Marrow Transplant 34(8): 693-702.

29. Sveikata A, Gumbrevicius G, Sestakauskas K, Kregzdyte R, Janulionis V, et al. (2014) Comparison of the pharmacokinetic and pharmacodynamic properties of two recombinant granulocyte colonystimulating factor formulations after single subcutaneous administration to healthy volunteers. Medicina (Kaunas) 50(3): 144-149.

30. McKenzie EC, Tornquist SJ, Gorman ME, Cebra CK, Payton ME (2008) Hematologic effects of subcutaneous administration of recombinant human granulocyte colony-stimulating factor (filgrastim) in healthy alpacas. Am J Vet Res 69(6): 770-776.

31. Simmons CP, Farrar JJ, Nguyen V, Wills B (2012) Dengue. N Engl J Med 366(15): 1423-1432.

32. Lee TH, Wong JG, Leo YS, Thein TL, Ee-Ling NG, et al. (2016) Potential Harm of Prophylactic Platelet Transfusion in Adult Dengue Patients. PloS Negl Trop Dis 10: e0004576.

33. Kaur R, Babita, Sama P (2014) Heamtological and Biochemical Changes in Dengue Fever. International Journal of Pharmaceutical Science Invention 3: 1-5.

34. Lee LK, Gan VC, Lee VJ, Tan AS, Leo YS, et al. (2012) Clinical relevance and discriminatory value of elevated liver aminotransferase levels for dengue severity. PloS Negl Trop Dis 6(6): e1676.

35. Dale DC, Bonilla MA, Davis MW, Nakanishi AM, Hammond WP, et al. (1993) A randomized controlled phase III trial of recombinant human granulocyte colony-stimulating factor (filgrastim) for treatment of severe chronic neutropenia. Blood 81(10): 24962502. 